\title{
A potential role for the paraventricular nucleus of the thalamus in mediating individual variation in Pavlovian conditioned responses
}

\author{
Joshua L. Haight ${ }^{1}$ and Shelly B. Flagel ${ }^{1,2,3}$ * \\ 1 Neuroscience Graduate Program, University of Michigan, Ann Arbor, MI, USA \\ ${ }^{2}$ Department of Psychiatry, University of Michigan, Ann Arbor, MI, USA \\ ${ }^{3}$ Molecular and Behavioral Neuroscience Institute, University of Michigan, Ann Arbor, MI, USA
}

Edited by:

Gavan McNally, University of New

South Wales, Australia

Reviewed by:

Kate M. Wassum, University of

California, Los Angeles, USA

Gilbert Jean Kirouac, University of

Manitoba, Canada

${ }^{*}$ Correspondence:

Shelly B. Flagel, Molecular and

Behavioral Neuroscience Institute,

University of Michigan, 205 Zina

Pitcher Place, Ann Arbor, MI 48109,

USA

e-mail: sflagel@umich.edu
There is ample evidence to suggest that the paraventricular nucleus of the thalamus (PVT) mediates cue-reward learning, especially as it relates to drug-seeking behavior. However, its exact role in these complex processes remains unknown. Here we will present and discuss data from our own laboratory which suggests that the PVT plays a role in multiple forms of stimulus-reward learning, and does so via distinct neurobiological systems. Using an animal model that captures individual variation in response to reward-associated cues, we are able to parse the incentive from the predictive properties of reward cues and to elucidate the neural circuitry underlying these different forms of cue-reward learning. When rats are exposed to a classical Pavlovian conditioning paradigm, wherein a cue predicts food reward, some rats, termed sign-trackers, approach and manipulate the cue upon its presentation. This behavior is indicative of attributing incentive salience to the cue. That is, the cue gains excessive control over behavior for sign-trackers. In contrast, other rats, termed goal-trackers, treat the cue as a mere predictor, and upon its presentation go to the location of reward delivery. Based on our own data utilizing this model, we hypothesize that the PVT represents a common node, but differentially regulates the sign- vs. goal-tracking response. We postulate that the PVT regulates sign-tracking behavior, or the attribution of incentive salience, via subcortical, dopamine-dependent mechanisms. In contrast, we propose that goal-tracking behavior, or the attribution of predictive value, is the product of "top-down" glutamatergic processing between the prelimbic cortex (PrL) and the PVT. Together, data from our laboratory and others support a role for the PVT in cue-motivated behaviors and suggest that it may be an important locus within the neural circuitry that goes awry in addiction and related disorders.

Keywords: paraventricular nucleus of the thalamus, sign-tracking, goal-tracking, cue-learning, motivated behavior, addiction, incentive salience, incentive stimuli

\section{INTRODUCTION}

Over the past few decades a large quantity of research has focused on elucidating the neurobiological mechanisms that contribute to addiction and related behaviors (for review see: Grace, 2000; Kelley and Berridge, 2002; Lüscher and Malenka, 2011; Everitt and Robbins, 2013; Nestler, 2014). The majority of this work has focused on the classic mesocorticolimbic reward circuitry, but the field is beginning to recognize the importance of structures outside of this system (e.g., Ikemoto, 2010). One such structure is the paraventricular nucleus of the thalamus (PVT), which has recently gained attention for its role in mediating cue-driven behaviors, especially as they relate to drug-seeking behavior and addiction (Martin-Fardon and Boutrel, 2012; James and Dayas, 2013; Browning et al., 2014). Although there is now sufficient evidence implicating the PVT in mediating responses to both food- and drug-associated cues, its exact role in these processes has yet to be discovered.

The PVT is a midline thalamic nucleus located at the interface between the limbic, cortical and motor circuits. The PVT receives a complex set of sub-cortical afferents from areas known to be involved in motivated behavior, including the hypothalamus, hippocampus, amygdala, locus coeruleus, periaqueductal grey, and dorsal raphe (Van der Werf et al., 2002; Vogt et al., 2008; Hsu and Price, 2009; Li and Kirouac, 2012). In addition to these sub-cortical elements, the PVT receives strong innervation from the medial prefrontal cortex, including the prelimbic, infralimbic, cingulate and dorsal peduncular cortices (Li and Kirouac, 2012). The densest set of afferents to the PVT appears to be from the prelimbic cortex (PrL; Li and Kirouac, 2012), an area recently shown to be a critical mediator of drug- and cue-motivated 
behaviors (Di Pietro et al., 2006; Di Ciano et al., 2007; Rocha and Kalivas, 2010). The efferents from the PVT are primarily glutamatergic, targeting both cortical and subcortical structures including the PrL, nucleus accumbens (NAc) shell and core, amygdala, hippocampus, and hypothalamus (Jones et al., 1989; Su and Bentivoglio, 1990; Van der Werf et al., 2002; Pinto et al., 2003; Li and Kirouac, 2008; Vertes and Hoover, 2008). Thus, the neuroanatomical positioning of the PVT is ideal for integrating information regarding environmental stimuli and internal states and translating it into motivated actions.

The first study to implicate the PVT as a potential mediator of motivated behavior surfaced almost 50 years ago when it was demonstrated that rats will self-stimulate intracranial electrodes placed in or near the PVT (Cooper and Taylor, 1967). These findings were later supported by Clavier and Gerfen (1982), who confirmed that the most consistent patterns of thalamic selfstimulation occurred when electrode placements were close to, or within the midline nuclei, which included the PVT. Since then, numerous studies have supported a role for the PVT in motivated behavior, specifically in response to discrete and contextual cues that have previously been paired with food and drug rewards. Here we review behavioral, pharmacological, and anatomical evidence supporting a role for the PVT in cue-motivated behaviors and, based on our own data, discuss a potential role for this structure in mediating specific aspects of cue-reward learning and Pavlovian conditioned approach behaviors.

\section{A ROLE FOR THE PARAVENTRICULAR NUCLEUS OF THE THALAMUS (PVT) IN REWARD PROCESSING AND CUE-MOTIVATED BEHAVIORS}

More than a decade following the intracranial self-stimulation studies (Cooper and Taylor, 1967; Clavier and Gerfen, 1982), the PVT was shown to play a role in psychoactive drug effects. Systemic administration of amphetamine and 3,4-methylenedioxy$\mathrm{N}$-methylamphetamine (MDMA) elicits an increase in neuronal activity in the PVT, as measured by $c$-fos (Deutch et al., 1995, 1998; Stephenson et al., 1999). Around this same time, a series of lesion studies sought to examine the role of the PVT in cocaineinduced behavioral sensitization. It was found that lesions of the PVT before (Young and Deutch, 1998), but not after (Pierce et al., 1997), a contextually conditioned regimen of repeated cocaine treatment attenuates the development of behavioral sensitization. These studies were the first to suggest that the PVT was important for the acquisition of the relationship between drugs and conditioned stimuli.

By this time it had been well established that motivated behaviors, such as behavioral sensitization, are regulated by a complex set of cortical, striatal, thalamic and limbic brain areas, known as the "motive circuit" (for review see Pierce and Kalivas, 1997). However, it wasn't until later that work by Ann Kelley et al. highlighted the PVT as an important component of this circuitry (Kelley et al., 2005a). In Kelley's model, the PVT is a critical interface between the limbic and motor circuitry, relaying information regarding arousal, environmental cues, energy needs, reward, and circadian rhythms from the hypothalamus to the striatum, including the NAc. Once in the striatum, this information is incorporated with other salient information from the ventral tegmental area (VTA) and prefrontal cortex, among other areas, and integrated with basal-ganglia motor output pathways to influence motivated behaviors. In support of this model, Kelley et al. demonstrated that exposure to a context previously paired with a highly palatable reward (chocolate Ensure) can induce robust cellular activation throughout many areas of the motive circuitry, including prefrontal cortical areas, the amygdala, NAc, and the PVT (Schiltz et al., 2005a, 2007). Interestingly, exposure to a context previously paired with nicotine administration also induces robust cellular activation in these areas (Schiltz et al., 2005b). This similar pattern of neuronal activation in response to both food and drug cues led Kelley et al. to postulate that "addictive drugs induce neuroadaptations in brain circuits normally subserving learning and memory for motivationally salient stimuli" (pg. 12, Kelley et al., 2005b), and the PVT appears to be a critical locus of these circuits.

Recent behavioral studies have built upon the initial studies by Kelley et al. (Kelley et al., 2005b; Schiltz et al., 2005a,b, 2007), further supporting the notion that the PVT is an important mediator of contextual cue-reward associations and addictionrelated behaviors (Martin-Fardon and Boutrel, 2012; James and Dayas, 2013). Johnson et al. demonstrated that exposure to a context previously paired with repeated experimenter administered cocaine injections increases levels of $c$-fos in the PVT (Johnson et al., 2010). Moreover, lesions or chemical inactivation of the PVT prevent reinstatement of "beer-seeking" behavior following exposure to the previously alcohol-paired context (Hamlin et al., 2009; Marchant et al., 2010). Additionally, the expression of cocaine-induced conditioned place preference is attenuated following inactivation of the PVT (Browning et al., 2014), further confirming a role for the PVT in contextual cue-reward processes.

Similar findings have been published with discrete rewardpaired cues. For example, repeated Pavlovian pairings of a discrete cue light with a water reward results in increased $c$-fos expression in the PVT relative to unpaired controls (Igelstrom et al., 2010). Likewise, exposure to a discrete odor cue previously associated with ethanol availability in a Pavlovian manner increases $c$-fos expression in the PVT (Dayas et al., 2008). C-fos is also elevated in the PVT following reinstatement of drug-seeking behaviors after exposure to ethanol- (Wedzony et al., 2003) or cocaine-associated (James et al., 2011) cues. Further, drug-seeking behavior can be disrupted by inactivation of the PVT, as James et al. demonstrated that a direct infusion of tetrodotoxin (a voltage-gated sodium channel antagonist) or the inhibitory peptide cocaineand amphetamine-regulated transcript (CART) into the PVT is able to attenuate cocaine-primed reinstatement (James et al., 2010). Taken together, these findings demonstrate a role for the PVT in the conditioned-effects of both discrete and contextual reward-associated cues, and drug-seeking behavior.

\section{PVT-DOPAMINE INTERACTIONS RELEVANT TO REWARD PROCESSING AND CUE-MOTIVATED BEHAVIORS}

A series of elegant studies have been published that further support a role for the PVT in motivated behaviors via its interactions with the dopamine system. Mesocorticolimbic dopamine transmission has long been known to play a role in cue- and 
drug-motivated behavior. Exposure to food or drug rewards, as well as reward-paired cues, elicits robust dopamine transmission in the NAc (for review see Baik, 2013). The PVT sends projections to the NAc core and, to a greater extent, the shell (Van der Werf et al., 2002; Li and Kirouac, 2008; Vertes and Hoover, 2008), and many of the these neurons are found in close proximity to tyrosine-hydroxylase positive (i.e., dopaminergic) axons (Pinto et al., 2003). This is one mechanism by which PVT activity can influence dopamine release in the ventral striatum. Another possibility is that the PVT affects accumbens dopamine activity by modulating presynaptic terminals. In support, Parsons et al. (2007) demonstrated that electrical excitation of the PVT elicits dopamine efflux independent of the VTA; and showed that these PVT-evoked responses were attenuated following intraaccumbens infusion of a glutamate receptor antagonist. Thus, glutamate release from PVT terminals appears to presynaptically regulate accumbens dopamine activity (Parsons et al., 2007). It has also been postulated that hypothalamic orexin neurons that project to the PVT are part of the sub-cortical system that drives dopamine levels in the ventral striatum (Kelley et al., 2005a). In support, in vivo administration of orexin-a peptide directly into the PVT has been shown to increase dopamine levels in the NAc (Choi et al., 2012). Thus, there are multiple ways in which the PVT can influence dopamine activity in the NAc, and in turn regulate motivated behaviors.

The PVT also receives sub-cortical input from dopaminergic neurons (Lindvall et al., 1984; García-Cabezas et al., 2009). Early biochemical evidence demonstrated that dopamine innervation of the PVT was, at least in part, coming from the VTA cell group in the ventromedial midbrain (Kizer et al., 1976). This was later supported by a retrograde tracing study showing that dopaminergic cells (i.e., tyrosine-hydroxylase positive) in the VTA projected to the PVT (Takada et al., 1990). However, it should be noted that tracing studies from other groups have not identified a circuit between the VTA and PVT (Cornwall and Phillipson, 1988; Chen and Su, 1990; Li and Kirouac, 2012). Alternatively, it has been suggested that dopaminergic innervation of the PVT arises primarily from the A11, A13 and A14 cell groups residing in the hypothalamus and periaqueductal gray (Lindvall et al., 1984). In support, it has since been demonstrated in monkeys that dopaminergic input to midline thalamic nuclei (PVT and centromedial nucleus combined) is coming from these cell groups, with the hypothalamus being the major source of input (SánchezGonzález et al., 2005). Retrograde transport from the PVT is evident in these same brain regions in rats (Chen and Su, 1990; Li and Kirouac, 2012), but there are cross-species differences in the pattern and density of dopaminergic innervation of the thalamus (García-Cabezas et al., 2009). Thus, further work is warranted to characterize the sources of dopaminergic input to the rat PVT.

Dopamine in the PVT presumably acts on dopamine D3 receptors, the primary dopamine receptor in the PVT (Mansour and Watson, 1995). We have recently confirmed the presence of D3 mRNA in the PVT using in situ hybridization, and remarkably, D3 expression is restricted to the PVT and not apparent in any of the surrounding thalamic nuclei (Figure 1). While the specific role of D3 activation in the PVT has yet to be examined, recent reports have demonstrated that systemic antagonism of D3

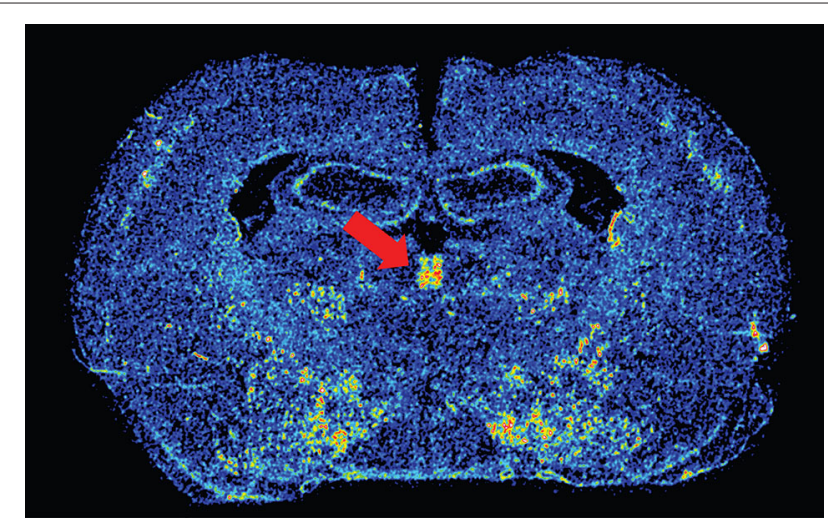

FIGURE 1 | Image of dopamine D3 receptor mRNA expression. Color-enhanced in situ hybridization image of D3 mRNA in the PVT (red arrow) in a coronal rat brain section. Approximate Bregma level is -2.28 (Paxinos and Watson, 2007).

receptors can block both drug- and cue-induced reinstatement of drug-seeking behaviors (Xi et al., 2006; Peng et al., 2009; Khaled et al., 2010; Higley et al., 2011; Rice et al., 2013). Interestingly, unpublished data from our own lab suggests that rats that are more susceptible to both drug- and cue-induced reinstatement have greater D3 mRNA expression in the PVT. Human imaging studies have also associated dopaminergic transmission in the thalamus with addiction-related behavior. Work by Volkow et al. has shown that methylphenidate administration in cocaine abusers leads to increased dopamine levels in the thalamus, which is positively correlated with reports of drug craving (Volkow et al., 1997). The resolution in human imaging studies does not allow one to distinguish the PVT from other thalamic nuclei, but these results are nonetheless interesting and relevant. Taken together, the literature reviewed above led us to postulate that the PVT influences cue- and reward-motivated behaviors by integrating information from sub-cortical systems, such as the orexin and dopamine neurotransmitter systems, and relaying that information to the ventral striatum, where it can impact NAc activity.

\section{EXPLOITING INDIVIDUAL VARIATION IN PAVLOVIAN CONDITIONED RESPONSES TO PARSE THE ROLE OF THE PARAVENTRICULAR NUCLEUS OF THE THALAMUS (PVT) IN CUE-REWARD LEARNING}

As summarized above, there is now sufficient evidence supporting the involvement of the PVT in motivated behavior and the processing of reward-associated cues. However, it is difficult to draw conclusions about the specific role of the PVT in these processes, since many of these studies are confounded by the fact that Pavlovian-conditioned reward cues can act not only as "predictors" of reward delivery, but can also come to act as "incentive" stimuli, capable of arousing complex emotional and motivational states (Stewart et al., 1984; Childress et al., 1993; Robinson and Berridge, 1993). It should be noted that here we are referring to incentive stimuli that have Pavlovian conditioned motivational properties, and not instrumental incentive value as described by Dickinson et al. (Balleine and Dickinson, 1998; 
Dickinson and Balleine, 2002). Pavlovian incentive stimuli have three fundamental properties: (1) they are attractive and elicit approach toward them, as in Pavlovian conditioned approach behavior; (2) they can reinforce the learning of new actions, acting as a conditioned reinforcer; and (3) they can energize ongoing instrumental actions, as in the Pavlovian instrumental transfer (PIT) effect (Estes, 1948; Lovibond, 1983; Berridge, 2001; Cardinal et al., 2002; Holmes et al., 2010). Until recently, it was thought that the conditional relationship between a cue and reward was sufficient to confer incentive motivational value to the cue. That is, if a cue attained predictive value and was capable of eliciting a conditioned response, then it was assumed that it also had the ability to act as an incentive stimulus. However, we have found that this is not the case (Robinson and Flagel, 2009).

Using an animal model, we have shown that there is considerable variation in the degree to which individuals will attribute predictive and incentive properties to reward-paired cues (Flagel et al., 2007; Robinson and Flagel, 2009; Meyer et al., 2012). When rats are exposed to a classical Pavlovian conditioning paradigm wherein an illuminated lever (conditioned stimulus) is repeatedly paired with delivery of a food reward (unconditioned stimulus), distinct conditioned responses emerge. Some rats, termed goaltrackers, attribute predictive value to the lever-cue, and promptly approach the location of reward delivery upon lever-cue presentation (Figure 2A). Other animals, called sign-trackers, not only attribute predictive value, but also attribute incentive salience to the lever-cue, and upon its presentation will approach and manipulate it (Figure 2B), even though no interaction with the lever is required for food delivery. Importantly, all of the animals, regardless of their phenotype, retrieve and eat all of the food pellets, and their behavior during the inter-trial intervals is the same and attenuates over training. Furthermore, if lever presentation is explicitly not paired with food delivery (i.e., unpaired conditions), neither conditioned response develops (Robinson and Flagel, 2009).

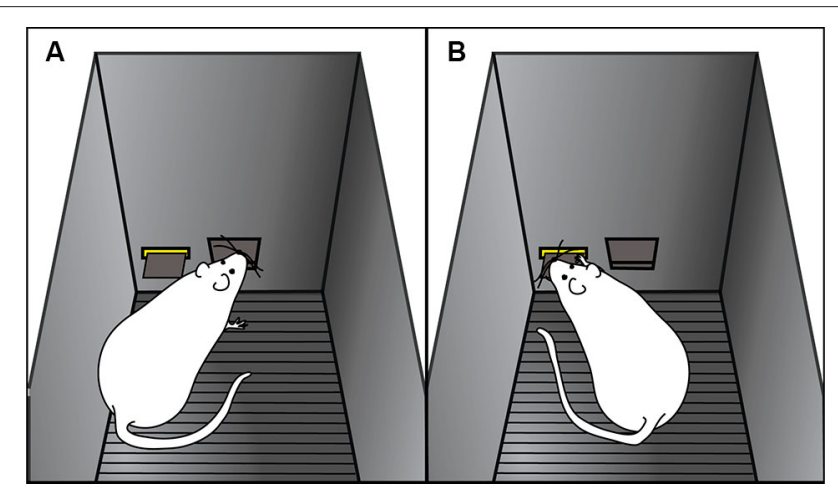

FIGURE 2 | Cartoon representation of goal-tracking and sign-tracking behaviors. Examples of (A) goal-tracking and (B) sign-tracking behaviors in response to lever-cue presentation during a Pavlovian conditioning session. (A) Goal-trackers approach the food cup (i.e., location of reward delivery) upon lever-cue presentation. (B) Sign-trackers approach the lever-cue during its presentation, even though no response is required for food delivery.
There is ample evidence supporting the notion that for signtrackers, but not goal-trackers, the lever-cue is attributed with incentive salience (e.g., Flagel et al., 2009; Meyer et al., 2012). For sign-trackers the cue itself is attractive and elicits approachindicative of the first quality of an incentive stimulus (Flagel et al., 2009). Further, for sign-trackers, the lever itself is desirable and acts as a more effective conditioned reinforcer relative to goal-trackers. That is, sign-trackers will respond more than goaltrackers for lever-cue presentation in the absence of food reward (Robinson and Flagel, 2009), demonstrating the second quality of an incentive stimulus. Evidence demonstrating individual variation in the third fundamental property of an incentive stimulus, i.e., general PIT, is lacking, perhaps due to the complex nature of the paradigm. However, there is evidence suggesting that reward cues arouse a conditioned motivational state to a greater extent in sign-trackers than goal-trackers (Saunders and Robinson, 2011, 2012; Saunders et al., 2013a). In sum, the lever-cue is a predictor of reward delivery for both sign- and goal-trackers, as it elicits a conditioned response in both and the responses are learned at the same rate; but only for sign-trackers does the cue serve as an incentive stimulus.

In support of the theory that the attribution of incentive salience to reward cues underlies addiction (Robinson and Berridge, 1993, 2001; Flagel et al., 2009), there is now evidence to suggest that sign-trackers are more likely to exhibit addiction-related behaviors (Saunders et al., 2013b; Robinson et al., 2014). Sign-trackers exhibit a greater propensity for psychomotor sensitization upon repeated treatment with cocaine (Flagel et al., 2008), a form of cocaine-induced plasticity that may contribute to the development of addiction. We have shown that rats who sign-track to food-associated cues do the same for drug-associated cues (Flagel et al., 2010; Yager and Robinson, 2013). Sign-trackers have also been reported to acquire cocaine self-administration more rapidly than goal-trackers (Beckmann et al., 2011). Further, cocaine-associated cues gain inordinate control over drug-taking behavior for sign-trackers, and these animals are more likely to exhibit reinstatement of drug-seeking behavior relative to goal-trackers, even in the face of adverse consequences (Saunders and Robinson, 2010, 2011; Saunders et al., 2013a). Sign-trackers are also more impulsive than goaltrackers, another trait associated with addiction liability in both animal models and humans (Belin et al., 2008; Ersche et al., 2010). Thus, individual differences in the propensity to attribute incentive salience to discrete food-paired cues confer vulnerability to addiction-related behaviors. It should be noted, however, that recent evidence suggests that goal-trackers may be more prone to attributing incentive motivational value to contextual stimuli (see Robinson et al., 2014), especially as they relate to drugs of abuse. These newly emerging findings provide further support for the notion that sign-trackers and goal-trackers process motivationally salient information in quite different ways (Flagel et al., 2011a,b; Robinson et al., 2014); and the PVT may play a central role in the underlying processes as it has previously been implicated the conditioned effects of both discrete and contextual reward-associated cues (e.g., Hamlin et al., 2009; James et al., 2011). 


\section{THE NEUROBIOLOGY UNDERLYING SIGN- AND GOAL-TRACKING BEHAVIOR}

Important findings surrounding the neurobiological mechanisms of cue-motivated behaviors have emerged from the signtracker/goal-tracker animal model. Exploiting these individual differences in stimulus-reward learning, we demonstrated that dopamine in the NAc core is necessary for both the learning and expression of a sign-tracking, but not goal-tracking, response (Flagel et al., 2011b; Saunders and Robinson, 2012; Saunders et al., 2013a). Thus, dopamine transmission is critical for the attribution of the incentive, but not necessarily the predictive, properties of reward cues. These findings underscore the fact that sign-tracking and goal-tracking are mediated by distinct neurobiological processes, with the former being dopamine-dependent and the latter dopamine-independent.

To further delineate the neural circuitry underlying the attribution of incentive vs. predictive value to reward cues, we sought to examine cue-induced neuronal activity in areas outside of the classic mesocorticolimbic dopamine circuitry. Outbred rats were characterized as sign-trackers vs. goal-trackers based on Pavlovian training sessions consisting of lever-cue presentations paired with food reward. After rats had learned their respective conditioned responses, they were presented with the lever in the absence of food reward to assess cue-induced expression of $c$-fos mRNA throughout the brain. Results showed that levels of $c$-fos mRNA were enhanced in the cortico-striatal-thalamic areas comprising the "motive circuit" (Kelley et al., 2005a) in sign-trackers relative to goal-trackers and controls, who received an equal number of lever-cue and food presentations but in an unpaired fashion (Flagel et al., 2011a). Thus, many parts of the motive circuit are engaged by the incentive, and not the predictive, properties of a discrete reward cue. Although sign-trackers exhibited enhanced cue-induced $c$-fos mRNA in all of the midline thalamic nuclei examined (i.e., central medial, intermediodorsal and PVT), the region with the most robust effect was the PVT (Flagel et al., 2011a). In response to cue presentation, sign-trackers exhibited almost twice as much $c$-fos expression in the PVT relative to goal-trackers. Importantly, goal-trackers did not significantly differ from the control group, suggesting that the PVT is highly engaged by cues attributed with incentive, but not predictive value. However, when we examined "functional connectivity" in sign-trackers vs. goal-trackers by identifying correlations in cueinduced $c$-fos mRNA between brain regions, a different picture emerged (Figure 3). Originally, this analysis included only brain regions in which there was a significant difference in cue-induced $c$-fos mRNA between sign-trackers and goal-trackers (Flagel et al., 2011a). Here, however, we have expanded this analysis to include all of the brain areas examined in order to get a more complete picture of network activity in the motive circuit.

In sign-trackers, cue induced $c$-fos mRNA expression was correlated between the thalamus and the NAc shell. Although this correlation was significant for multiple thalamic nuclei, the strongest was a negative correlation $(r=-0.9)$ between the PVT and the NAc shell. It should be noted that, with this analysis, the direction of the correlation is uninformative, since the type of cell (e.g., inhibitory or excitatory) in which the $c$-fos is expressed remains unknown. Regardless, this finding further supports a role for the PVT in dopamine-dependent, sub-cortical processing of the sign-tracking response. For goal-trackers, cue-induced $c$ fos mRNA was correlated between the prefrontal cortex and the PVT. Of particular interest is the significant correlation $(r=0.7)$ expressed between the PVT and the PrL, since the densest set of afferents to the PVT comes from the PrL (Li and Kirouac, 2012). Interestingly, there were no significant correlations between the PrL and other thalamic nuclei. There was also evidence of corticostriatal communication in goal-trackers, which was not present in sign-trackers. These distinct patterns of connectivity highlight the extent to which different neural systems are engaged when a cue is attributed with incentive vs. predictive value and highlight a potential role for the PVT in these learning processes.

\section{THE ROLE OF THE PARAVENTRICULAR NUCLEUS OF THE THALAMUS (PVT) IN MEDIATING SIGN- VS. GOAL-TRACKING BEHAVIORS}

The discovery that PVT activity is increased following exposure to a reward-associated cue in sign-trackers, but not goal-trackers, suggests a specific role for the PVT in the attribution of incentive salience. We hypothesize that dopaminergic and orexinergic subcortical projections to the PVT, coupled with the dense PVT efferents to the ventral striatum (Li and Kirouac, 2008), may be mediating this process. The mesolimbic dopamine system has long been known to be active in response to reward cues, and the lateral hypothalamus, which contains PVT-projecting orexin neurons, has recently been recognized for a similar role (Choi et al., 2010). It is possible therefore, that exposure to a reward-paired cue elicits robust activity in both dopaminergic and orexinergic projections to the PVT, which could result in increased excitation in PVT neurons. This increased activity in the PVT could ultimately lead to an increase in dopamine activity in the NAc, and may do so to a greater extent than VTA-NAc transmission alone. Presumably, activity in each of these pathways that mediate dopamine release in the NAc are enhanced to a greater extent in sign-trackers than goal-trackers in response to reward cues.

Our previous work has demonstrated that sign-tracking behavior is dependent on dopamine transmission in the NAc core (Flagel et al., 2011b; Saunders and Robinson, 2012); but the role of dopamine in the NAc shell in these behaviors has yet to be investigated. Importantly, the NAc core and shell send direct projections to one another via medium spiny neurons and interneurons (van Dongen et al., 2005). The NAc shell also sends projections directly to the VTA (Nauta et al., 1978; Heimer et al., 1991), and these projections heavily overlap with VTA cells that in turn project back to the NAc core (Haber et al., 2000). Therefore, there are both direct and indirect pathways in which the NAc shell can influence activity in the NAc core. Further, while ample evidence supports a role for the NAc core in cue-reward processing, recent evidence has demonstrated a potentially similar role for the NAc shell (Blaiss and Janak, 2009; Grimm et al., 2011; Peciña and Berridge, 2013). In relation, we found enhanced cueinduced $c$-fos activity in both the core and shell in sign-trackers relative to goal-trackers (Flagel et al., 2011a). Thus, the specific involvement of the NAc core vs. shell in cue-motivated behaviors is not yet entirely clear. We suspect, however, that PVT projections to the NAc affect activity in both the core and the shell and it is, at 
A

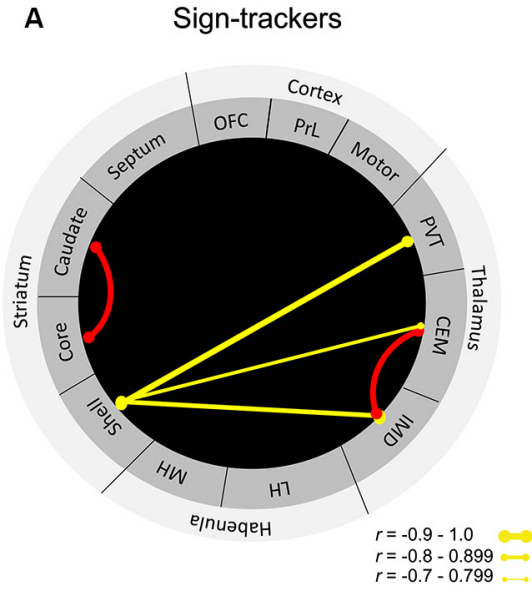

FIGURE 3 | "Functional connectivity" in sign-trackers and goal-trackers. Illustration of significantly correlated levels of c-fos mRNA expression between brain regions for $(\mathbf{A})$ sign-trackers and (B) goal-trackers. Red lines are indicative of a significant positive correlation and yellow lines represent negative correlations. The thicker the line, the

\section{B Goal-trackers}

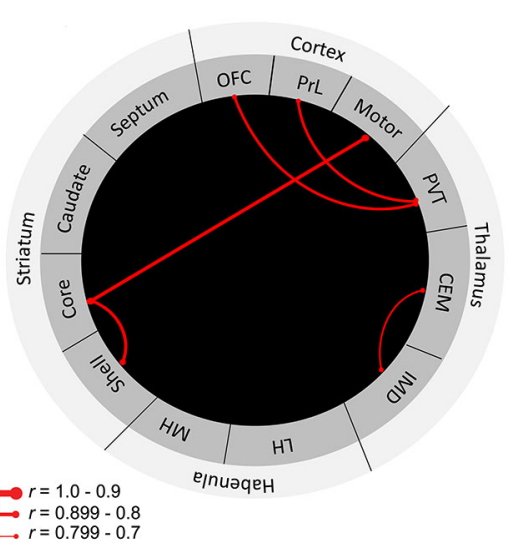

stronger the correlation. Abbreviations: OFC, orbitofrontal cortex; PrL, prelimbic cortex, PVT, paraventricular nucleus of the thalamus; CEM, centromedial nucleus of the thalamus; IMD, intermediodorsal nucleus of the thalamus; LH, lateral habenula; $\mathrm{MH}$, medial habenula. Adapted from Flagel et al. (2011a). least in part, via this circuit that the PVT regulates sign-tracking behavior. Based on existing data (Flagel et al., 2011b; Saunders and Robinson, 2012), it is difficult to know whether these effects would occur via modulation of tonic or phasic dopamine release, or both. Nonetheless, this orexin/dopamine-PVT-NAc pathway warrants further investigation as it could play a critical role in incentive salience attribution and prove to be a novel target for the treatment of addiction-related behaviors.

Perhaps more surprising than the discovery of PVT involvement in incentive salience attribution is the new data reported here that PVT and PrL activity is correlated in goal-trackers, but not sign-trackers, following cue presentation. The PrL is important for regulating goal-directed behavior (Balleine and Dickinson, 1998), and has recently been thought to represent a "cognitive-control" mechanism capable of inhibiting conditioned responding to cues (Jonkman et al., 2009; Kober et al., 2010; Mihindou et al., 2013). Indeed, we have shown that goal-trackers exhibit more self-control, as they are found to be less impulsive than sign-trackers (Flagel et al., 2010; Lovic et al., 2011), and perform better on a prefrontal-dependent sustained-attention task (Paolone et al., 2013). Moreover, both the goal-tracking response and cognitively-mediated learning processes are known to be dopamine independent (Dickinson and Balleine, 2002; Flagel et al., 2011b; Saunders and Robinson, 2012; Saunders et al., 2013a). Together, these findings led us to the hypothesis that goal-trackers utilize the discrete reward cue as an informational stimulus which results in the attribution of predictive (but not incentive) value to the cue, via a "top-down" (e.g., PrL-PVT) cognitive learning strategy. In consideration of the circuitry proposed above for sign-trackers, it is possible that for goal-trackers PrL input to the PVT is suppressing the subcortical (i.e., orexinergic/dopaminergic) signaling induced by the reward cue, preventing an increase in accumbens dopamine levels, and thereby preventing the attribution of incentive salience to the cue. For example, the PVT shows dense expression of group II metabotropic glutamate receptors, and agonism of these receptors leads to hyperpolarization of post-synaptic PVT neurons (Hermes and Renaud, 2011). PrL glutamatergic activity at these receptors could therefore result in the suppression of sub-cortical orexin and dopamine signaling at the level of the PVT. Alternatively, PrL input to the PVT could be exciting local GABAergic interneurons, leading to an overall inhibition of the structure, and thereby inhibiting accumbens dopamine activity.

In sum, we are proposing that the PVT is a critical node wherein integration of sub-cortical and cortical inputs can influence the propensity to attribute incentive vs. predictive qualities to discrete reward cues (Figure 4). In support, preliminary data from our lab suggests that lesions of the PVT differentially alter the sign- vs. goal-tracking response (unpublished data). Specifically, lesions of the PVT appear to enhance sign-tracking behavior and attenuate goal-tracking behavior. Interestingly, these effects were only apparent in the sign-tracking response after it had been acquired. That is, lesions of the PVT seemed to enhance the vigor of the sign-tracking response, but only during peak performance. In contrast, PVT lesions attenuate both the acquisition and peak performance of the goal-tracking response. It is important to note that these lesions were performed prior to Pavlovian training, and due to the nondescript nature of lesion studies we cannot at this time draw strong conclusions regarding the cell-type or circuitry contributing to the observed effects. Although the proposed mechanisms by which the PVT regulates the attribution of incentive vs. predictive value to reward cues are purely speculative and perhaps oversimplified at this point, our own data and those of others support the notion that the PVT is critical to multiple forms of stimulus-reward learning that are relevant to addiction (Flagel et al., 2009; Robinson et al., 2014). 


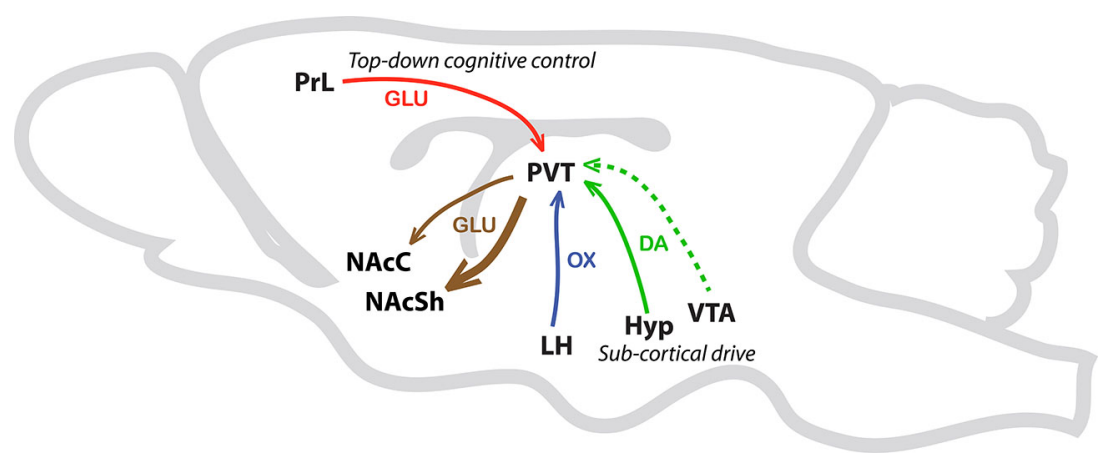

FIGURE 4 | Schematic illustrating afferents and efferents of interest in the PVT. This simplified schematic illustrates PVT afferents and efferents that are potentially involved in Pavlovian conditioned approach behavior. The solid green arrow represents sub-cortical dopamine inputs from the hypothalamus (Hyp). The dashed green line represents less dense dopaminergic input from the ventral tegmental area (VTA). The blue arrow represents orexin (OX) input from the lateral hypothalamus (LH) and the red arrow represents glutamatergic (GLU) projections from the prelimbic cortex (PrL) to the PVT. Efferent pathways from the PVT to the nucleus accumbens shell (NAcSh), and to a lesser extent the nucleus accumbens core (NAcC), are represented with brown arrows.

\section{CONCLUSION}

Based on anatomical, pharmacological, and behavioral evidence, the PVT appears to play an important role in mediating cuemotivated behaviors. Recent data from our laboratory suggests that the role of the PVT in motivated behavior lies in processing both the predictive and incentive properties of reward cues. It is hypothesized that the PVT is a critical regulator in biasing an individual towards either dopamine-dependent (signtracking) or dopamine-independent (goal-tracking) behaviors. In this model, sign-tracking behavior is mediated by a "subcortical drive" involving dopaminergic and orexinergic input to the PVT; while "top-down" cognitive control of behavior, in the form of dense glutamatergic PrL innervation of the PVT, underlies goal-tracking behavior. Ongoing studies using optogenetics and Designer Receptors Exclusively Activated by Designer Drugs (DREADD) receptor technology will allow us to further delineate the role of this nucleus and related circuitry in sign- and goal-tracking behaviors. Despite the fact that the PVT has begun to emerge as a major player in motivated behaviors, cue learning, and associated psychopathologies such as addiction, we have only begun to understand this complex nucleus. Further investigations into the function of the PVT, as well as its efferents and afferents, are warranted before we can begin to fully comprehend the neural circuitry underlying motivated behavior.

\section{ACKNOWLEDGMENTS}

The authors would like to thank Drs. Terry E. Robinson, Maria Waselus, and Stanley J. Watson for reading earlier versions of this manuscript and providing helpful comments. We would also like to thank Katie Long for her help in preparing the figures and Kurt Fraser who provided technical assistance for the unpublished studies that were referenced.

\section{REFERENCES}

Baik, J.-H. (2013). Dopamine signaling in reward-related behaviors. Front. Neural Circuits 7:152. doi: 10.3389/fncir.2013.00152
Balleine, B. W., and Dickinson, A. (1998). Goal-directed instrumental action: contingency and incentive learning and their cortical substrates. Neuropharmacology 37, 407-419. doi: 10.1016/s0028-3908(98)00033-1

Beckmann, J. S., Marusich, J. A., Gipson, C. D., and Bardo, M. T. (2011). Novelty seeking, incentive salience and acquisition of cocaine self-administration in the rat. Behav. Brain Res. 216, 159-165. doi: 10.1016/j.bbr.2010.07.022

Belin, D., Mar, A. C., Dalley, J. W., Robbins, T. W., and Everitt, B. J. (2008). High impulsivity predicts the switch to compulsive cocaine-taking. Science 320, $1352-$ 1355. doi: 10.1126/science. 1158136

Berridge, K. C. (2001). "Reward learning: reinforcement, incentives and expectations," in Psychology of Learning and Motivation, ed D. Medin (Waltham, MA: Academic Press), 223-278.

Blaiss, C. A., and Janak, P. H. (2009). The nucleus accumbens core and shell are critical for the expression, but not the consolidation, of Pavlovian conditioned approach. Behav. Brain Res. 200, 22-32. doi: 10.1016/j.bbr.2008. 12.024

Browning, J. R., Jansen, H. T., and Sorg, B. A. (2014). Inactivation of the paraventricular thalamus abolishes the expression of cocaine conditioned place preference in rats. Drug Alcohol Depend. 134, 387-390. doi: 10.1016/j. drugalcdep.2013.09.021

Cardinal, R. N., Parkinson, J. A., Lachenal, G., Halkerston, K. M., Rudarakanchana, N., Hall, J., et al. (2002). Effects of selective excitotoxic lesions of the nucleus accumbens core, anterior cingulate cortex, and central nucleus of the amygdala on autoshaping performance in rats. Behav. Neurosci. 116, 553-567. doi: 10. 1037/0735-7044.116.4.553

Chen, S., and Su, H.-S. (1990). Afferent connections of the thalamic paraventricular and parataenial nuclei in the rat-a retrograde tracing study with iontophoretic application of Fluoro-Gold. Brain Res. 522, 1-6. doi: 10.1016/00068993(90)91570-7

Childress, A. R., Hole, A. V., Ehrman, R. N., Robbins, S. J., Mclellan, A. T., and O'brien, C. P. (1993). Cue reactivity and cue reactivity interventions in drug dependence. NIDA Res. Monogr. 137, 73-95.

Choi, D. L., Davis, J. F., Fitzgerald, M. E., and Benoit, S. C. (2010). The role of orexin-A in food motivation, reward-based feeding behavior and foodinduced neuronal activation in rats. Neuroscience 167, 11-20. doi: 10.1016/j. neuroscience.2010.02.002

Choi, D. L., Davis, J. F., Magrisso, I. J., Fitzgerald, M. E., Lipton, J. W., and Benoit, S. C. (2012). Orexin signaling in the paraventricular thalamic nucleus modulates mesolimbic dopamine and hedonic feeding in the rat. Neuroscience 210, $243-$ 248. doi: 10.1016/j.neuroscience.2012.02.036

Clavier, R., and Gerfen, C. (1982). Intracranial self-stimulation in the thalamus of the rat. Brain Res. Bull. 8, 353-358. doi: 10.1016/0361-9230(82)90072-7

Cooper, R., and Taylor, L. (1967). Thalamic reticular system and central gray: self stimulation. Science 156, 102-103. doi: 10.1126/science.156.3771.102 
Cornwall, J., and Phillipson, O. T. (1988). Afferent projections to the dorsal thalamus of the rat as shown by retrograde lectin transport. II. The midline nuclei. Brain Res. Bull. 21, 147-161. doi: 10.1016/0361-9230(88)90227-4

Dayas, C. V., Mcgranahan, T. M., Martin-Fardon, R., and Weiss, F. (2008). Stimuli linked to ethanol availability activate hypothalamic CART and orexin neurons in a reinstatement model of relapse. Biol. Psychiatry 63, 152-157. doi: 10.1016/j. biopsych.2007.02.002

Deutch, A. Y., Bubser, M., and Young, C. D. (1998). Psychostimulant-induced Fos protein expression in the thalamic paraventricualr nucleus. J. Neurosci. 18, 10680-10687.

Deutch, A. Y., Ongur, D., and Duman, R. S. (1995). Antipsychotic drugs induce Fos protein in the thalamic paraventricular nucleus: a novel locus of antipsychotic drug action. Neuroscience 66, 337-346. doi: 10.1016/0306-4522(94)00 571-1

Di Ciano, P., Benham-Hermetz, J., Fogg, A. P., and Osborne, G. E. C. (2007). Role of the prelimbic cortex in the acquisition, re-acquisition or persistence of responding for a drug-paired conditioned reinforcer. Neuroscience 150, 291-298. doi: 10.1016/j.neuroscience.2007.09.016

Di Pietro, N. C., Black, Y. D., and Kantak, K. M. (2006). Context-dependent prefrontal cortex regulation of cocaine self-administration and reinstatement behaviors in rats. Eur. J. Neurosci. 24, 3285-3298. doi: 10.1111/j.1460-9568.2006. 05193.x

Dickinson, A., and Balleine, B. W. (2002). "The role of learning in the operation of motivational systems," in Stevens' Book of Experimental Psychology: Learning, Motivation and Emotion, eds H. Pashler and R. Gallistel 3rd Edn. (New York: John Wiley and Sons), 497-533.

Ersche, K. D., Turton, A. J., Pradhan, S., Bullmore, E. T., and Robbins, T. W. (2010). Drug addiction endophenotypes: impulsive versus sensation-seeking personality traits. Biol. Psychiatry 68, 770-773. doi: 10.1016/j.biopsych.2010. 06.015

Estes, W. K. (1948). Discriminative conditioning. II. Effects of a Pavlovian conditioned stimulus upon a subsequently established operant response. J. Exp. Psychol. 38, 173-177. doi: 10.1037/h0057525

Everitt, B. J., and Robbins, T. W. (2013). From the ventral to the dorsal striatum: devolving views of their roles in drug addiction. Neurosci. Biobehav. Rev. 37, 1946-1954. doi: 10.1016/j.neubiorev.2013.02.010

Flagel, S. B., Akil, H., and Robinson, T. E. (2009). Individual differences in the attribution of incentive salience to reward-related cues: implications for addiction. Neuropharmacology 56(Suppl. 1), 139-148. doi: 10.1016/j.neuropharm.2008. 06.027

Flagel, S. B., Cameron, C. M., Pickup, K. N., Watson, S. J., Akil, H., and Robinson, T. E. (2011a). A food predictive cue must be attributed with incentive salience for it to induce c-fos mRNA expression in cortico-striatal-thalamic brain regions. Neuroscience 196, 80-96. doi: 10.1016/j.neuroscience.2011.09.004

Flagel, S. B., Clark, J. J., Robinson, T. E., Mayo, L., Czuj, A., Willuhn, I., et al. (2011b). A selective role for dopamine in stimulus-reward learning. Nature 469, 53-57. doi: 10.1038/nature09588

Flagel, S. B., Robinson, T. E., Clark, J. J., Clinton, S. M., Watson, S. J., Seeman, P., et al. (2010). An animal model of genetic vulnerability to behavioral disinhibition and responsiveness to reward-related cues: implications for addiction. Neuropsychopharmacology 35, 388-400. doi: 10.1038/npp.2009.142

Flagel, S. B., Watson, S. J., Akil, H., and Robinson, T. E. (2008). Individual differences in the attribution of incentive salience to a reward-related cue: influence on cocaine sensitization. Behav. Brain Res. 186, 48-56. doi: 10.1016/j. bbr.2007.07.022

Flagel, S. B., Watson, S. J., Robinson, T. E., and Akil, H. (2007). Individual differences in the propensity to approach signals vs goals promote different adaptations in the dopamine system of rats. Psychopharmacology (Berl) 191, 599-607. doi: 10.1007/s00213-006-0535-8

Grace, A. A. (2000). The tonic/phasic model of dopamine system regulation and its implications for understanding alcohol and psychostimulant craving. Addiction 95, S119-S128. doi: 10.1046/j.1360-0443.95.8s2.1.x

García-Cabezas, M. Á., Martínez-Sánchez, P., Sánchez-González, M. Á., Garzón, M., and Cavada, C. (2009). Dopamine innervation in the thalamus: monkey versus rat. Cereb. Cortex 19, 424-434. doi: 10.1093/cercor/bhn093

Grimm, J., Harkness, J., Ratliff, C., Barnes, J., North, K., and Collins, S. (2011) Effects of systemic or nucleus accumbens-directed dopamine D1 receptor antagonism on sucrose seeking in rats. Psychopharmacology (Berl) 216, 219-233. doi: 10.1007/s00213-011-2210-y
Haber, S. N., Fudge, J. L., and Mcfarland, N. R. (2000). Striatonigrostriatal pathways in primates form an ascending spiral from the shell to the dorsolateral striatum. J. Neurosci. 20, 2369-2382. doi: 10.0270-6474/00/202369-14

Hamlin, A. S., Clemens, K. J., Choi, E. A., and Mcnally, G. P. (2009). Paraventricular thalamus mediates context-induced reinstatement (renewal) of extinguished reward seeking. Eur. J. Neurosci. 29, 802-812. doi: 10.1111/j.1460-9568.2009. 06623.x

Heimer, L., Zahm, D. S., Churchill, L., Kalivas, P. W., and Wohltmann, C. (1991). Specificity in the projection patterns of accumbal core and shell in the rat. Neuroscience 41, 89-125. doi: 10.1016/0306-4522(91)90202-y

Hermes, M. L., and Renaud, L. P. (2011). Postsynaptic and presynaptic group II metabotropic glutamate receptor activation reduces neuronal excitability in rat midline paraventricular thalamic nucleus. J. Pharmacol. Exp. Ther. 336, 840-849. doi: 10.1124/jpet.110.176149

Higley, A. E., Kiefer, S. W., Li, X., Gaál, J., Xi, Z.-X., and Gardner, E. L. (2011). Dopamine D3 receptor antagonist SB-277011A inhibits methamphetamine selfadministration and methamphetamine-induced reinstatement of drug-seeking in rats. Eur. J. Pharmacol. 659, 187-192. doi: 10.1016/j.ejphar.2011.02.046

Holmes, N. M., Marchand, A. R., and Coutureau, E. (2010). Pavlovian to instrumental transfer: a neurobehavioural perspective. Neurosci. Biobehav. Rev. 34, 1277-1295. doi: 10.1016/j.neubiorev.2010.03.007

Hsu, D. T., and Price, J. L. (2009). Paraventricular thalamic nucleus: subcortical connections and innervation by serotonin, orexin and corticotropin-releasing hormone in macaque monkeys. J. Comp. Neurol. 512, 825-848. doi: 10.1002/cne. 21934

Igelstrom, K. M., Herbison, A. E., and Hyland, B. I. (2010). Enhanced c-Fos expression in superior colliculus, paraventricular thalamus and septum during learning of cue-reward association. Neuroscience 168, 706-714. doi: 10.1016/j. neuroscience.2010.04.018

Ikemoto, S. (2010). Brain reward circuitry beyond the mesolimbic dopamine system: a neurobiological theory. Neurosci. Biobehav. Rev. 35, 129-150. doi: 10. 1016/j.neubiorev.2010.02.001

James, M. H., Charnley, J. L., Flynn, J. R., Smith, D. W., and Dayas, C. V. (2011). Propensity to 'relapse' following exposure to cocaine cues is associated with the recruitment of specific thalamic and epithalamic nuclei. Neuroscience 199, 235 242. doi: 10.1016/j.neuroscience.2011.09.047

James, M. H., Charnley, J. L., Jones, E., Levi, E. M., Yeoh, J. W., Flynn, J. R., et al. (2010). Cocaine- and amphetamine-regulated transcript (CART) signaling within the paraventricular thalamus modulates cocaine-seeking behaviour. PLoS One 5:e12980. doi: 10.1371/journal.pone.0012980

James, M. H., and Dayas, C. V. (2013). What about me...? The PVT: a role for the paraventricular thalamus (PVT) in drug-seeking behaviour. Front. Behav. Neurosci. 7:18. doi: 10.3389/fnbeh.2013.00018

Johnson, Z. V., Revis, A. A., Burdick, M. A., and Rhodes, J. S. (2010). A similar pattern of neuronal Fos activation in 10 brain regions following exposure to reward- or aversion-associated contextual cues in mice. Physiol. Behav. 99, 412418. doi: 10.1016/j.physbeh.2009.12.013

Jones, M. W., Kilpatrick, I. C., and Phillipson, O. T. (1989). Regulation of dopamine function in the nucleus accumbens of the rat by the thalamic paraventricular nucleus and adjacent midline nuclei. Exp. Brain Res. 76, 572-580. doi: 10. 1007/bf00248914

Jonkman, S., Mar, A. C., Dickinson, A., Robbins, T. W., and Everitt, B. J. (2009). The rat prelimbic cortex mediates inhibitory response control but not the consolidation of instrumental learning. Behav. Neurosci. 123, 875-885. doi: 10. 1037/a0016330

Kelley, A. E., Baldo, B. A., and Pratt, W. E. (2005a). A proposed hypothalamicthalamic-striatal axis for the integration of energy balance, arousal and food reward. J. Comp. Neurol. 493, 72-85. doi: 10.1002/cne.20769

Kelley, A. E., and Berridge, K. C. (2002). The neuroscience of natural rewards: relevance to addictive drugs. J. Neurosci. 22, 3306-3311. doi: 10.0270-6474/02/ 223306-06

Kelley, A. E., Schiltz, C. A., and Landry, C. F. (2005b). Neural systems recruited by drug- and food-related cues: studies of gene activation in corticolimbic regions. Physiol. Behav. 86, 11-14. doi: 10.1016/j.physbeh.2005.06.018

Khaled, M. A. T. M., Farid Araki, K., Li, B., Coen, K. M., Marinelli, P. W., Varga, J., et al. (2010). The selective dopamine D3 receptor antagonist SB 277011-A, but not the partial agonist BP 897, blocks cue-induced reinstatement of nicotine-seeking. Int. J. Neuropsychopharmacol. 13, 181-190. doi: 10. $1017 /$ s1461145709991064 
Kizer, J. S., Palkovits, M., and Brownstein, M. J. (1976). The projections of the A8, A9 and A10 dopaminergic cell bodies: evidence for a nigral-hypothalamicmedian eminence dopaminergic pathway. Brain Res. 108, 363-370. doi: 10. 1016/0006-8993(76)90192-x

Kober, H., Mende-Siedlecki, P., Kross, E. F., Weber, J., Mischel, W., Hart, C. L., et al. (2010). Prefrontal-striatal pathway underlies cognitive regulation of craving. Proc. Natl. Acad. Sci. U S A 107, 14811-14816. doi: 10.1073/pnas.1007779107

Li, S., and Kirouac, G. J. (2008). Projections from the paraventricular nucleus of the thalamus to the forebrain, with special emphasis on the extended amygdala. $J$. Comp. Neurol. 506, 263-287. doi: 10.1002/cne.21502

Li, S., and Kirouac, G. J. (2012). Sources of inputs to the anterior and posterior aspects of the paraventricular nucleus of the thalamus. Brain Struct. Funct. 217, 257-273. doi: 10.1007/s00429-011-0360-7

Lindvall, O., Björklund, A., and Skagerberg, G. (1984). Selective histochemical demonstration of dopamine terminal systems in rat di- and telecephalon: new evidence for dopaminergic innervation of hypothalamic neurosecretory nuclei. Brain Res. 306, 19-30. doi: 10.1016/0006-8993(84)90352-4

Lovibond, P. F. (1983). Facilitation of instrumental behavior by a Pavlovian appetitive conditioned stimulus. J. Exp. Psychol. Anim. Behav. Process. 9, 225-247. doi: 10.1037/0097-7403.9.3.225

Lovic, V., Saunders, B. T., Yager, L. M., and Robinson, T. E. (2011). Rats prone to attribute incentive salience to reward cues are also prone to impulsive action. Behav. Brain Res. 223, 255-261. doi: 10.1016/j.bbr.2011.04.006

Lüscher, C., and Malenka, R. C. (2011). Drug-evoked synaptic plasticity in addiction: from molecular changes to circuit remodeling. Neuron 69, 650-663. doi: 10.1016/j.neuron.2011.01.017

Mansour, A., and Watson, S. J. (1995). "Dopamine receptor expression in the central nervous system," in Psychopharmacology: The Fifth Generation, eds F. E. Bloom and D. J. Kupfer (New York: Raven Press, Ltd.), 207-219.

Marchant, N. J., Furlong, T. M., and Mcnally, G. P. (2010). Medial dorsal hypothalamus mediates the inhibition of reward seeking after extinction. J. Neurosci. 30, 14102-14115. doi: 10.1523/jneurosci.4079-10.2010

Martin-Fardon, R., and Boutrel, B. (2012). Orexin/hypocretin (Orx/Hcrt) transmission and drug-seeking behavior: is the paraventricular nucleus of the thalamus (PVT) part of the drug seeking circuitry? Front. Behav. Neurosci. 6:75. doi: 10.3389/fnbeh.2012.00075

Meyer, P. J., Lovic, V., Saunders, B. T., Yager, L. M., Flagel, S. B., Morrow, J. D., et al. (2012). Quantifying individual variation in the propensity to attribute incentive salience to reward cues. PLoS One 7:e38987. doi: 10.1371/journal.pone.0038987

Mihindou, C., Guillem, K., Navailles, S., Vouillac, C., and Ahmed, S. H. (2013). Discriminative inhibitory control of cocaine seeking involves the prelimbic prefrontal cortex. Biol. Psychiatry 73, 271-279. doi: 10.1016/j.biopsych.2012.08. 011

Nauta, W. J. H., Smith, G. P., Faull, R. L. M., and Domesick, V. B. (1978). Efferent connections and nigral afferents of the nucleus accumbens septi in the rat. Neuroscience 3, 385-401. doi: 10.1016/0306-4522(78)90041-6

Nestler, E. J. (2014). Epigenetic mechanisms of drug addiction. Neuropharmacology 76(Pt. B), 259-268. doi: 10.1016/j.neuropharm.2013.04.004

Paolone, G., Angelakos, C. C., Meyer, P. J., Robinson, T. E., and Sarter, M. (2013). Cholinergic control over attention in rats prone to attribute incentive salience to reward cues. J. Neurosci. 33, 8321-8335. doi: 10.1523/jneurosci.0709-13.2013

Parsons, M. P., Li, S., and Kirouac, G. J. (2007). Functional and anatomical connection between the paraventricular nucleus of the thalamus and dopamine fibers of the nucleus accumbens. J. Comp. Neurol. 500, 1050-1063. doi: $10.1002 / \mathrm{cne} .21224$

Paxinos, G., and Watson, C. (2007). The Rat Brain in Stereotaxic Coordinates. Burlingame, MA: Academic Press.

Peciña, S., and Berridge, K. C. (2013). Dopamine or opioid stimulation of nucleus accumbens similarly amplify cue-triggered 'wanting' for reward: entire core and medial shell mapped as substrates for PIT enhancement. Eur. J. Neurosci. 37, 1529-1540. doi: 10.1111/ejn.12174

Peng, X.-Q., Ashby, C. R., Jr., Spiller, K., Li, X., Li, J., Thomasson, N., et al. (2009). The preferential dopamine D3 receptor antagonist S33138 inhibits cocaine reward and cocaine-triggered relapse to drug-seeking behavior in rats. Neuropharmacology 56, 752-760. doi: 10.1016/j.neuropharm.2008.12.007

Pierce, R. C., and Kalivas, P. W. (1997). A circuitry model of the expression of behavioral sensitization to amphetamine-like psychostimulants. Brain Res. Brain Res. Rev. 25, 192-216. doi: 10.1016/s0165-0173(97)00021-0
Pierce, R. C., Reeder, D. C., Hicks, J., Morgan, Z. R., and Kalivas, P. W. (1997). Ibotenic acid lesions of the dorsal prefrontal cortex disrupt the expression of behavioral sensitization to cocaine. Neuroscience 82, 1103-1114. doi: 10 . 1016/s0306-4522(97)00366-7

Pinto, A., Jankowski, M., and Sesack, S. R. (2003). Projections from the paraventricular nucleus of the thalamus to the rat prefrontal cortex and nucleus accumbens shell: ultrastructural characteristics and spatial relationships with dopamine afferents. J. Comp. Neurol. 459, 142-155. doi: 10.1002/cne.10596

Rice, O. V., Heidbreder, C. A., Gardner, E. L., Schonhar, C. D., and Ashby, C. R. (2013). The selective D3 receptor antagonist SB-277011A attenuates morphinetriggered reactivation of expression of cocaine-induced conditioned place preference. Synapse 67, 469-475. doi: 10.1002/syn.21653

Robinson, T. E., and Berridge, K. C. (1993). The neural basis of drug craving: an incentive-sensitization theory of addiction. Brain Res. Brain Res. Rev. 18, 247291. doi: 10.1016/0165-0173(93)90013-p

Robinson, T. E., and Berridge, K. C. (2001). Incentive-sensitization and addiction. Addiction 96, 103-114. doi: 10.1046/j.1360-0443.2001.9611038.x

Robinson, T. E., and Flagel, S. B. (2009). Dissociating the predictive and incentive motivational properties of reward-related cues through the study of individual differences. Biol. Psychiatry 65, 869-873. doi: 10.1016/j.biopsych.2008.09.006

Robinson, T. E., Yager, L. M., Cogan, E. S., and Saunders, B. T. (2014). On the motivational properties of reward cues: individual differences. Neuropharmacology 76(Pt. B), 450-459. doi: 10.1016/j.neuropharm.2013.05.040

Rocha, A., and Kalivas, P. W. (2010). Role of the prefrontal cortex and nucleus accumbens in reinstating methamphetamine seeking. Eur. J. Neurosci. 31, $903-$ 909. doi: 10.1111/j.1460-9568.2010.07134.x

Sánchez-González, M. Á., García-Cabezas, M. Á., Rico, B., and Cavada, C. (2005). The primate thalamus is a key target for brain dopamine. J. Neurosci. 25, 60766083. doi: 10.1523/jneurosci.0968-05.2005

Saunders, B. T., and Robinson, T. E. (2010). A cocaine cue acts as an incentive stimulus in some but not others: implications for addiction. Biol. Psychiatry 67, 730-736. doi: 10.1016/j.biopsych.2009.11.015

Saunders, B. T., and Robinson, T. E. (2011). Individual variation in the motivational properties of cocaine. Neuropsychopharmacology 36, 1668-1676. doi: 10. 1038/npp.2011.48

Saunders, B. T., and Robinson, T. E. (2012). The role of dopamine in the accumbens core in the expression of Pavlovian-conditioned responses. Eur. J. Neurosci. 36, 2521-2532. doi: 10.1111/j.1460-9568.2012.08217.x

Saunders, B. T., Yager, L. M., and Robinson, T. E. (2013a). Cue-evoked cocaine "craving": role of dopamine in the accumbens core. J. Neurosci. 33, 1398914000. doi: 10.1523/jneurosci.0450-13.2013

Saunders, B. T., Yager, L. M., and Robinson, T. E. (2013b). Preclinical studies shed light on individual variation in addiction vulnerability. Neuropsychopharmacology 38, 249-250. doi: 10.1038/npp.2012.161

Schiltz, C., Bremer, Q., Landry, C., and Kelley, A. (2007). Food-associated cues alter forebrain functional connectivity as assessed with immediate early gene and proenkephalin expression. BMC Biol. 5:16. doi: 10.1186/1741-7007-5-16

Schiltz, C., Kelley, A., and Landry, C. (2005a). Exposure to cues associated with palatable food increases immediate-early gene (IEG) mRNA and proenkephalin (PENK) premRNA expression in the rat brain. Neuropsychopharmacology 30, S213. doi: 10.1038/sj.npp.1300970

Schiltz, C. A., Kelley, A. E., and Landry, C. F. (2005b). Contextual cues associated with nicotine administration increase arc mRNA expression in corticolimbic areas of the rat brain. Eur. J. Neurosci. 21, 1703-1711. doi: 10.1111/j.1460-9568. 2005.04001.x

Stephenson, C. P., Hunt, G. E., Topple, A. N., and Mcgregor, I. S. (1999). The distribution of 3,4-methylenedioxymethamphetamine "Ecstasy"-induced $c$-fos expression in rat brain. Neuroscience 92, 1011-1023. doi: 10.1016/s03064522(99)00049-4

Stewart, J., De Wit, H., and Eikelboom, R. (1984). Role of unconditioned and conditioned drug effects in the self-administration of opiates and stimulants. Psychol. Rev. 91, 251-268. doi: 10.1037/0033-295x.91.2.251

Su, H.-S., and Bentivoglio, M. (1990). Thalamic midline cell populations projecting to the nucleus accumbens, amygdala and hippocampus in the rat. J. Comp. Neurol. 297, 582-593. doi: 10.1002/cne.902970410

Takada, M., Campbell, K. J., Moriizumi, T., and Hattori, T. (1990). On the origin of the dopaminergic innervation of the paraventricular thalamic nucleus. Neurosci. Lett. 115, 33-36. doi: 10.1016/0304-3940(90)90513-9 
Van der Werf, Y. D., Witter, M. P., and Groenewegen, H. J. (2002). The intralaminar and midline nuclei of the thalamus. Anatomical and functional evidence for participation in processes of arousal and awareness. Brain Res. Brain Res. Rev. 39, 107-140. doi: 10.1016/s0165-0173(02)00181-9

van Dongen, Y. C., Deniau, J. M., Pennartz, C. M. A., Galis-De Graaf, Y., Voorn, P., Thierry, A. M., et al. (2005). Anatomical evidence for direct connections between the shell and core subregions of the rat nucleus accumbens. Neuroscience 136, 1049-1071. doi: 10.1016/j.neuroscience.2005.08.050

Vertes, R. P., and Hoover, W. B. (2008). Projections of the paraventricular and paratenial nuclei of the dorsal midline thalamus in the rat. J. Comp. Neurol. 508, 212-237. doi: 10.1002/cne.21679

Vogt, B., Hof, P., Friedman, D., Sikes, R., and Vogt, L. (2008). Norepinephrinergic afferents and cytology of the macaque monkey midline, mediodorsal and intralaminar thalamic nuclei. Brain Struct. Funct. 212, 465-479. doi: 10. 1007/s00429-008-0178-0

Volkow, N. D., Wang, G.-J., Fischman, M. W., Foltin, R. W., Fowler, J. S., Abumrad, N. N., et al. (1997). Relationship between subjective effects of cocaine and dopamine transporter occupancy. Nature 386, 827-830. doi: 10.1038/38 $6827 \mathrm{a} 0$

Wedzony, K., Koros, E., Czyrak, A., Chocyk, A., Czepiel, K., Fijal, K., et al. (2003). Different pattern of brain c-Fos expression following re-exposure to ethanol or sucrose self-administration environment. Naunyn Schmiedebergs Arch. Pharmacol. 368, 331-341. doi: 10.1007/s00210-003-0811-7

Xi, Z.-X., Newman, A. H., Gilbert, J. G., Pak, A. C., Peng, X.-Q., Ashby, C. R. Jr., et al. (2006). The novel dopamine D3 receptor antagonist NGB 2904 inhibits cocaine's rewarding effects and cocaine-induced reinstatement of drug-seeking behavior in rats. Neuropsychopharmacology 31, 1393-1405. doi: 10.1038/sj.npp. 1300912

Yager, L. M., and Robinson, T. E. (2013). A classically conditioned cocaine cue acquires greater control over motivated behavior in rats prone to attribute incentive salience to a food cue. Psychopharmacology (Berl) 226, 217-228. doi: 10.1007/s00213-012-2890-y

Young, C. D., and Deutch, A. Y. (1998). The effects of thalamic paraventricular nucleus lesions on cocaine-induced locomotor activity and sensitization. Pharmacol. Biochem. Behav. 60, 753-758. doi: 10.1016/s0091-3057(98)00051-3

Conflict of Interest Statement: The authors declare that the research was conducted in the absence of any commercial or financial relationships that could be construed as a potential conflict of interest.

Received: 13 January 2014; paper pending published: 28 January 2014; accepted: 24 February 2014; published online: 14 March 2014.

Citation: Haight JL and Flagel SB (2014) A potential role for the paraventricular nucleus of the thalamus in mediating individual variation in Pavlovian conditioned responses. Front. Behav. Neurosci. 8:79. doi: 10.3389/fnbeh.2014.00079

This article was submitted to the journal Frontiers in Behavioral Neuroscience

Copyright (c) 2014 Haight and Flagel. This is an open-access article distributed under the terms of the Creative Commons Attribution License (CC BY). The use, distribution or reproduction in other forums is permitted, provided the original author(s) or licensor are credited and that the original publication in this journal is cited, in accordance with accepted academic practice. No use, distribution or reproduction is permitted which does not comply with these terms. 